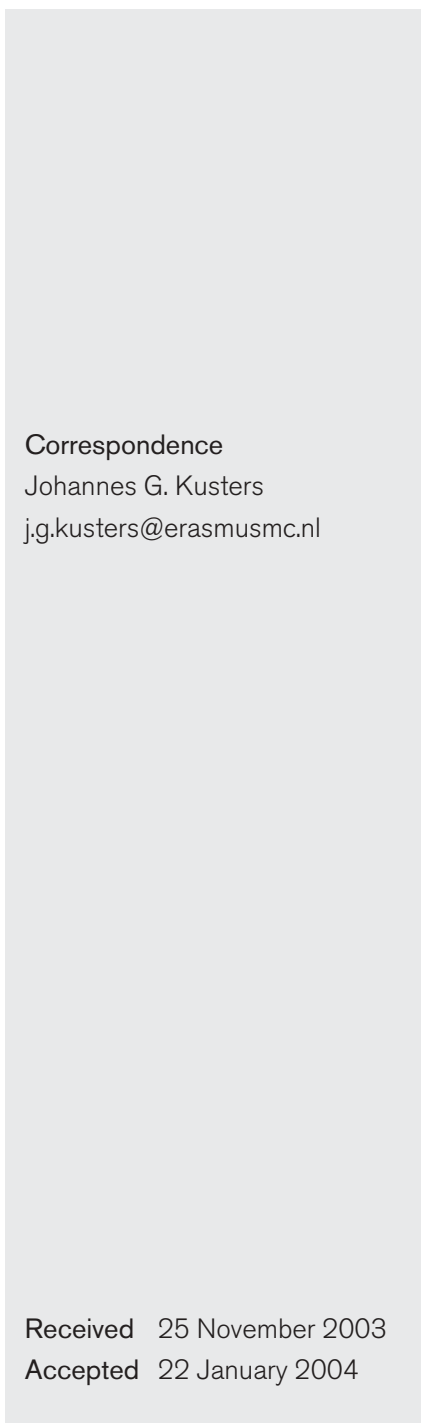

\title{
Role of the Helicobacter pylori outer-membrane proteins AlpA and AlpB in colonization of the guinea pig stomach
}

\author{
Ramon de Jonge, ${ }^{1,2}$ Zarmina Durrani, ${ }^{3}$ Sjoerd G. Rijpkema, ${ }^{3}$ \\ Ernst J. Kuipers, ${ }^{1}$ Arnoud H. M. van Vliet ${ }^{1}$ \\ and Johannes G. Kusters ${ }^{1}$ \\ ${ }^{1}$ Department of Gastroenterology and Hepatology, Erasmus MC-University Medical Center \\ Rotterdam, Rotterdam, the Netherlands \\ ${ }^{2}$ Department of Gastroenterology, VU University Medical Center, Amsterdam, the Netherlands \\ ${ }^{3}$ Division of Bacteriology, National Institute for Biological Standards and Control, Potters Bar, UK
}

\begin{abstract}
The human gastric pathogen Helicobacter pylori expresses several putative outer-membrane proteins (OMPs), but the role of individual OMPs in colonization of the stomach by $H$. pylori is still poorly understood. The role of four such OMPs (AlpA, AlpB, OipA and HopZ) in a guinea pig model of $H$. pylori infection has been investigated. Single alpA, alpB, hop $Z$ and oip $A$ isogenic mutants were constructed in the guinea pig-adapted, wild-type H. pylori strain GP15. Guinea pigs were inoculated intragastrically with the wild-type strain, single mutants or a mixture of the wild-type and a single mutant in a $1: 1$ ratio. Three weeks after infection, $H$. pylori could be isolated from stomach sections of all animals that were infected with the wild-type, the hopZ mutant or the oipA mutant, but from only five of nine $(P=0.18)$ and one of seven $(P=0.02)$ animals that were infected with the alpA or alp $B$ mutants, respectively. The hopZ and oip $A$ mutants colonized the majority of animals that were inoculated with the strain mixture, whereas alp $A$ and $a / p B$ mutants could not be isolated from animals that were infected with the strain mixture $(P<0.01)$. Specific $\lg G$ antibody responses were observed in all animals that were infected with either the wild-type or a mutant, but lgG levels were lower in animals that were infected with either the $a / p A$ or the alpB mutants, compared to the wildtype strain $(P<0.05)$. In conclusion, absence of AlpA or AlpB is a serious disadvantage for colonization of the stomach by H. pylori.
\end{abstract}

\section{INTRODUCTION}

The human pathogen Helicobacter pylori persistently colonizes the gastric mucosa of more than half of the world's population. Colonization by $H$. pylori always results in active gastritis and is associated with the development of peptic ulcer disease, gastric adenocarcinoma and mucosa-associated lymphoid tissue lymphoma (Kuipers, 1997). Several $H$. pylori factors are commonly believed to be required for efficient, lifelong colonization of the gastric mucosa, including urease activity (Eaton \& Krakowka, 1994), motility by polar sheathed flagella (Eaton et al., 1996) and adherence to gastric epithelial cells (Hessey et al., 1990; Guruge et al., 1998; Yamaoka et al., 2002). Adherence to gastric

Abbreviations: $\mathrm{Kan}^{\mathrm{R}}$, kanamycin-resistance; OMP, outer-membrane protein. epithelial cells protects $H$. pylori against peristalsis and mucosal shedding and warrants access to nutrients that are released from damaged epithelium.

It appears that mucosal adhesion of $H$. pylori is associated directly with mucosal damage (Hessey et al., 1990; Guruge et al., 1998). Adhesion of $H$. pylori is mediated in part via specific adhesins, such as the sialyl Lewis ${ }^{\mathrm{x}}$-binding protein SabA (Mahdavi et al., 2002) and the Lewis ${ }^{\mathrm{b}}$ blood group antigen-binding protein BabA (Boren et al., 1993; Ilver et al., 1998). However, the $H$. pylori genome also encodes several other outer-membrane proteins (OMPs) that have been associated with adhesion, including OipA, HopZ, AlpA and AlpB (Alm et al., 1999; Evans \& Evans, 2000; Tomb et al., 1997). The role of these individual OMPs in adhesion of $H$. pylori is still relatively poorly understood. $H$. pylori strains with reduced expression of OipA, SabA, SabB and HopZ displayed limited colonization of the murine stomach when two or more of these OMPs were not expressed, due to phase 
variation (Yamaoka et al., 2002). AlpA and AlpB may function as adhesins, as isogenic alp $A$ and alp $B$ mutants displayed reduced adherence to human gastric tissue and gastric epithelial cells when tested in vitro (Odenbreit et al., 1999, 2002a).

Several animal models have been employed for study of $H$. pylori infection, using non-human primates, gerbils, mice and guinea pigs (Marchetti et al., 1995; Dubois et al., 1996; Hirayama et al., 1996; Guruge et al., 1998; Shomer et al., 1998). The guinea pig model for $H$. pylori infection has some attractive features, such as ease of husbandry and animal size. The guinea pig stomach has several features in common with the human stomach that are lacking in the mouse model, such as sterility and the production of IL8 (Shomer et al., 1998; Sturegard et al., 1998).

In this study, we have employed the guinea pig model of H. pylori infection to characterize the role of the OMPs AlpA, AlpB, OipA and HopZ in colonization of the stomach by H. pylori. Isogenic mutants in the alp $A, \operatorname{alp} B$, oip $A$ and hop $Z$ genes were created in the guinea pig-adapted $H$. pylori strain GP15 and used to infect guinea pigs with either single mutants or $1: 1$ mixtures of the wild-type strain and a single mutant. Three weeks after inoculation, colonization efficiency and local and systemic antibody responses were measured. We thus observed that both AlpA and AlpB make important contributions to gastric colonization by $H$. pylori.

\section{METHODS}

Bacterial strain and culture conditions. The guinea pig-adapted H. pylori strain GP15 (Rijpkema et al., 2001) and isogenic mutants in the four OMP-encoding genes were cultured routinely on Columbia agar medium plates that were supplemented with $5 \%$ defibrinated horse blood or $7 \%$ saponin-lysed horse blood and Dent selective supplement (Oxoid), further referred to as Dent plates. H. pylori was grown routinely for $72-96 \mathrm{~h}$ at $37{ }^{\circ} \mathrm{C}$ in an atmosphere of $5 \% \mathrm{O}_{2}, 10 \%$ $\mathrm{CO}_{2}$ and $85 \% \mathrm{~N}_{2}$. Broth cultures were grown in Brucella broth that was supplemented with $3 \%$ newborn calf serum (Gibco-BRL) and Dent supplement (Oxoid). When appropriate, $H$. pylori growth media were supplemented with kanamycin to a final concentration of $20 \mu \mathrm{g} \mathrm{ml}^{-1}$.

Construction of site-directed mutants and recombinant DNA techniques. Standard recombinant DNA techniques were performed as described by Sambrook et al. (1989). Plasmid pJMK30 (van Vliet et al., 1998) was used as the source of the kanamycin-resistance $\left(\operatorname{Kan}^{\mathrm{R}}\right)$ cassette. PCR fragments that contained an internal part of the alpA, alpB, oipA or hopZ genes were amplified by PCR using the primers listed in Table 1 and were subsequently cloned into pGEM-T Easy vector (Promega). The $\mathrm{Kan}^{\mathrm{R}}$ cassette was inserted into the unique Eco47III, SmaI, HindIII and SmaI sites in the alpA, alpB, oipA and hopZ genes, respectively. The resulting plasmids were used to transform $H$. pylori strain GP15 by natural transformation, as described previously (Bijlsma et al., 1999). Correct integration of the $\mathrm{Kan}^{\mathrm{R}}$ cassette was verified by restriction analysis and PCR amplification, using primers located outside the amplified region that was used for creation of the respective mutants (Table 1).

Animal experiments and serology. Infection experiments were performed essentially as described previously (Rijpkema et al., 2001).

Table 1. Oligonucleotide primers used in this study

\begin{tabular}{|c|c|c|c|c|}
\hline \multirow[t]{2}{*}{ Gene } & \multicolumn{2}{|c|}{ Gene number in strain ${ }^{\star}$} & \multirow[t]{2}{*}{ Primer $\dagger$} & \multirow[t]{2}{*}{ Sequence $\left(5^{\prime} \rightarrow 3^{\prime}\right)$} \\
\hline & 26695 & J99 & & \\
\hline \multirow{4}{*}{ alpA } & HР0912 & JHP0848 & AlpA-F1 & ACAACACCGGAGGCAATGTC \\
\hline & & & AlpA-R1 & GATAGGAGCGGCTAAAGATG \\
\hline & & & AlpA-F2 & AGGAGCATCCCATTGATAAG \\
\hline & & & AlpA-R2 & TGCCTTGCACCAAACCTGTA \\
\hline \multirow[t]{4}{*}{ alpB } & HР0913 & JHP0849 & AlpB-F1 & CTTTATGACCTTTGGGTATG \\
\hline & & & AlpB-R1 & GAGCGGCTAAACACATTGTA \\
\hline & & & AlpB-F2 & AGCCTAGCGTTAAGCTTGAG \\
\hline & & & AlpB-R2 & TTCATGCGTAACCCCACATG \\
\hline \multirow[t]{4}{*}{ oipA } & HР0638 & JHP0581 & OipA-F1 & CACGCTGAAAGGAACGGATT \\
\hline & & & OipA-R1 & AAGATAAACGCTCACCACTC \\
\hline & & & OipA-F2 & GATTGATTAAAGGCGTGGTG \\
\hline & & & OipA-R2 & CTATCAAGGAGAGCGAAAGA \\
\hline \multirow[t]{4}{*}{ hopZ } & НР0009 & JHP0007 & HopZ-F1 & CСTTACACCTCTGCTGATAG \\
\hline & & & HopZ-R1 & ATCTTCACGCCTAGTTCCAC \\
\hline & & & HopZ-F2 & AGTTTAGCGCCGTTACTAGC \\
\hline & & & HopZ-R2 & GAGTTGAGTGCCTAGCAAAG \\
\hline
\end{tabular}

${ }^{\star}$ Primer sequences were based on the complete genome sequences of H. pylori strains 26695 and J99 (Tomb et al., 1997; Alm et al., 1999).

$\dagger F 1 / R 1$ primer combinations were used to amplify gene fragments for insertion of the $\mathrm{Kan}^{\mathrm{R}}$ cassette; F2/R2 primer combinations are located outside the F1/R1 fragment and were used to verify correct integration of the $\operatorname{Kan}^{\mathrm{R}}$ cassette into the $H$. pylori genome. 
Female Dunkin-Hartley guinea pigs (200-300 g) were housed on grids and fed with water and food ad libitum. All animal experiments were performed according to the guidelines of the Home Office (1986) and were approved by the local ethics committee. Guinea pigs were fasted overnight before being given an intragastric dose of either wild-type H. pylori strain GP15, its isogenic mutants or a mixture of both at a ratio of $1: 1$. Either two or three doses of approximately $10^{9}$ c.f.u. H. pylori per dose were given to animals intragastrically on alternate days (Rijpkema et al., 2001). Animals were dissected 21 days after inoculation and serum, bile and gastric mucosa of the antral region were collected. The stomach was rinsed briefly with PBS and a small section of the gastric antrum was streaked out on Dent plates to detect wild-type $H$. pylori, and on kanamycin-supplemented Dent plates to detect $H$. pylori mutants. Colonies were identified as $H$. pylori by morphology and urease activity. Antibodies were extracted from antral tissue as described previously (Bergquist et al., 2000). Specific IgG and IgA were detected by ELISA, using plates that were coated with $H$. pylori GP15 outermembrane fraction, as described previously (Rijpkema et al., 2001; Durrani \& Rijpkema, 2003).

Statistical analysis. The Mann-Whitney $U$ test for non-parametric data was used to compare differences between experimental groups. All data are presented as mean $\pm \mathrm{SD}$. $P$ values of $<0.05$ were considered to be significant.

\section{RESULTS AND DISCUSSION}

\section{Characterization of $\boldsymbol{H}$. pylori OMP mutants}

To evaluate the contribution of the AlpA, AlpB, OipA and HopZ proteins in gastric colonization by $H$. pylori, isogenic mutants in the respective genes were created in $H$. pylori strain GP15. Parts of the $\operatorname{alpA,}$ alpB, oipA and hopZ genes were amplified by PCR, using primer F1/R1 combinations (Table 1), and cloned in an E. coli vector; the coding region of the genes was interrupted by the insertion of a $\mathrm{Kan}^{\mathrm{R}}$ cassette. Subsequently, the mutated genes were used for allelic exchange after natural transformation of $H$. pylori and kanamycin-resistant colonies were selected. Correct replacement of the wild-type gene by the mutated copy was confirmed by PCR using F2/R2 primer combinations (Table 1). Absence of one of these OMPs did not affect growth under standard growth conditions, as the growth rate of the mutants was similar to that of the wild-type strain. There was also no significant difference between the mutants and the wild-type strain in the ability to induce IL8 production in gastric epithelial cells (data not shown), confirming the previously reported phenotype of the mutants (Ando et al., 2002; Odenbreit et al., 2002b).

\section{Effect of OMP gene mutation on gastric colonization by $\boldsymbol{H}$. pylori in guinea pigs}

The role of the AlpA, AlpB, OipA and HopZ proteins in gastric colonization in vivo was evaluated by using the guinea pig model for H. pylori infection (Rijpkema et al., 2001; Durrani \& Rijpkema, 2003). Ability to colonize was first assessed by comparing single infections with the wild-type strain GP15 and single isogenic mutants in two independent infection experiments. The results of these experiments are shown in Fig. 1a. Wild-type H. pylori GP15 and the oipA and
hopZ mutants colonized all inoculated guinea pigs, confirming previous observations made by using a mouse model of H. pylori infection (Yamaoka et al., 2002). The alpA mutant could only be isolated from five of nine inoculated guinea pigs, but this difference was not significant $(P=0 \cdot 18)$. In contrast, the alpB mutant could only be isolated from one of seven inoculated animals $(P=0 \cdot 02)$.

The attenuating effect of the OMP mutations was further defined by using competitive infection experiments with wild-type GP15 and each of the single OMP mutants in a $1: 1$ ratio. Wild-type and OMP mutants could be distinguished by the kanamycin resistance of mutant cells. The wild-type strain was isolated from all inoculated animals, irrespective of the OMP mutant included in the $1: 1$ mixture (Fig. 1b). The hopZ and oipA mutants could also be isolated from more than half of the inoculated animals, indicating that neither mutant had a major selective disadvantage when compared to the wild-type strain. In contrast, the alpA and $a l p B$

(a)

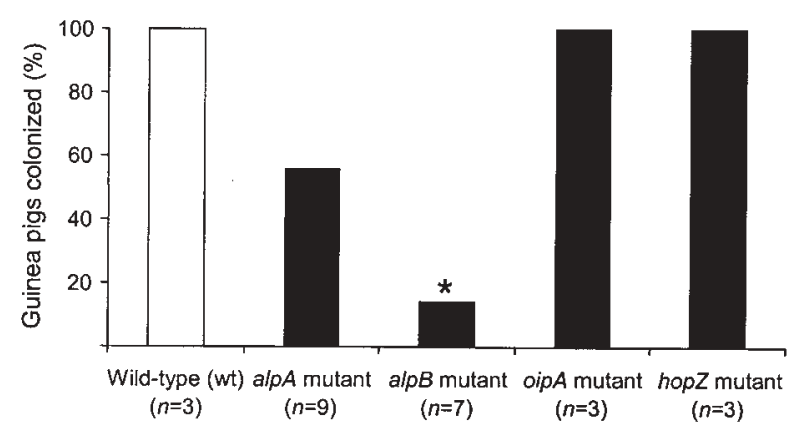

H. pylori strain

(b)

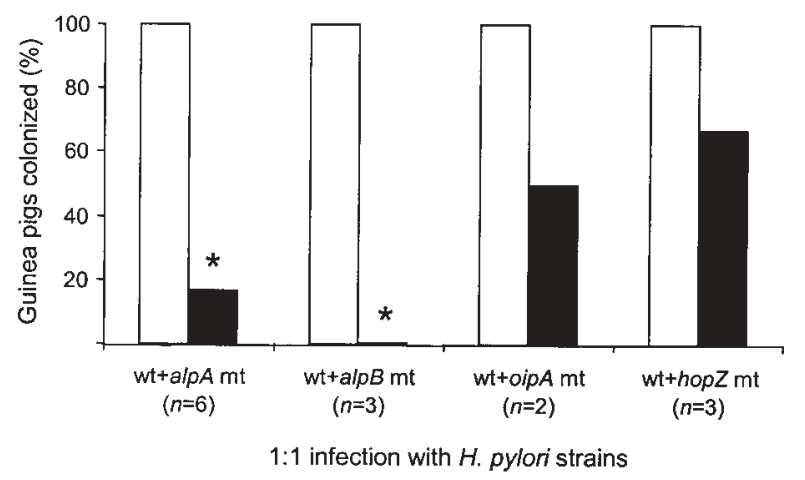

Fig. 1. Colonization of guinea pigs by wild-type $H$. pylori GP15 and isogenic OMP mutants. (a) Single inoculation of either wild-type $H$. pylori strain GP15 (empty bar) or alpA, alpB, oipA or hopZ mutants (filled bars). (b) Results of competitive infection experiments, using $1: 1$ mixtures of wild-type GP15 (empty bars) and OMP mutants (filled bars). Asterisks indicate a significant difference between the number of animals colonized after inoculation with $H$. pylori OMP mutants, compared to infection by the wild-type strain GP15 $(P<0.05)$. 
mutants could only be isolated from one of six $(P=0 \cdot 01)$ and none of three $(P=0.03)$ infected guinea pigs, respectively.

\section{Antibody responses to infection by OMP mutants}

Differential effects of the OMP mutations were apparent in the antibody response in serum, as well as in locally produced antral mucosa IgG and bile IgA (Fig. 2). Serum IgG and antral IgG antibody responses were significantly lower in animals that were inoculated with the alp $B$ mutant, compared to the wild-type strain $(P=0 \cdot 01$ in both cases). Serum IgG, but not antral mucosal IgG, was also decreased in animals that were inoculated with the alpA mutant $(P=0 \cdot 01)$. This finding may be due to incomplete colonization (56\%) of animals that were inoculated with the alpA mutant (Fig. 1). There was no difference between the levels of specific IgG antibodies in animals that were inoculated with wild-type GP15 and the oipA and hopZ mutants. Interestingly, bile IgA levels were significantly lower in animals that were inoculated with the alpB, oipA and hopZ mutants ( $P$ values of $0.01,0.03$ and 0.03 , respectively), but not with the alpA mutant $(P=0 \cdot 12)$ (Fig. $2)$. We currently have no explanation for this unexpected difference between the IgG and IgA responses with the oipA and hop $Z$ mutants. Antibody responses in animals that were infected with 1:1 ratios of wild-type and single OMP mutants were similar to those infected with the wild-type GP15 strain (data not shown), which is not surprising in view of the total colonization by the wild-type strain.

\section{DISCUSSION}

Our findings extend previous observations (made in vitro) that the alp operon (which consists of alpA and $a l p B$ ) is involved in adherence to gastric epithelial cells and gastric tissue sections (Odenbreit et al., 1999, 2002a) and demonstrate that the Alp OMPs contribute significantly to successful infection of guinea pigs by $H$. pylori. The marked decrease in colonization efficiency of alp $A$ and alpB mutants can be attributed to a deficiency of the mutants to adhere to gastric epithelial cells in vivo, as was demonstrated previously in vitro by using gastric biopsies (Odenbreit et al., 2002a). As a consequence of inactivation of these two OMP-encoding genes, $H$. pylori can probably be removed easily from the stomach by gastric emptying and may therefore be unable to persist at the epithelial surface and cause inflammation of gastric mucosa.

The alpA gene is transcribed in vivo in human gastric tissues, as well as in murine gastric tissues, in the first 3 months of infection (Rokbi et al., 2001), indicating that the Alp proteins play an active role in establishing and maintaining gastric colonization. Transcription of alpA was tenfold higher $1 \mathrm{~h}$ post-infection than 1 week post-infection; by comparison, transcription of ureA and 16S rRNA varied much less over time (Rokbi et al., 2001). The role of AlpA and AlpB in the early stages of infection may make them important candidates for a prophylactic vaccine. Indeed, vaccination studies in mice have indicated that an AlpA vaccine reduced bacterial load significantly when given as a prophylaxis, but was ineffective when given as a therapeutic treatment (Sanchez et al., 2001).

\section{Conclusion}

In this study, it is demonstrated that the H. pylori OMPs AlpB and, to a lesser extent, AlpA, are required for gastric colonization of the guinea pig stomach, most probably by mediating adherence to gastric epithelial cells. Further work is required to determine whether alp $A$ or alp $B$ mutants may serve as an attenuated live vaccine. Characterization of the receptors and functions of individual $H$. pylori OMP molecules may provide further insights into mechanisms that are essential for $H$. pylori colonization of human gastric mucosa.

\section{ACKNOWLEDGEMENTS}

The authors would like to thank Stefan Odenbreit for helpful discussions. This study was supported by a grant from the Universitair Stimulerings Fonds (USF) of the Vrije Universiteit, Amsterdam, to E.J.K.

\section{REFERENCES}

Alm, R. A., Ling, L.-S. L., Moir, D. T. \& 20 other authors (1999). Genomicsequence comparison of two unrelated isolates of the human gastric pathogen Helicobacter pylori. Nature 397, 176-180.

Ando, T., Peek, R. M., Jr, Lee, Y.-C., Krishna, U., Kusugami, K. \& Blaser,

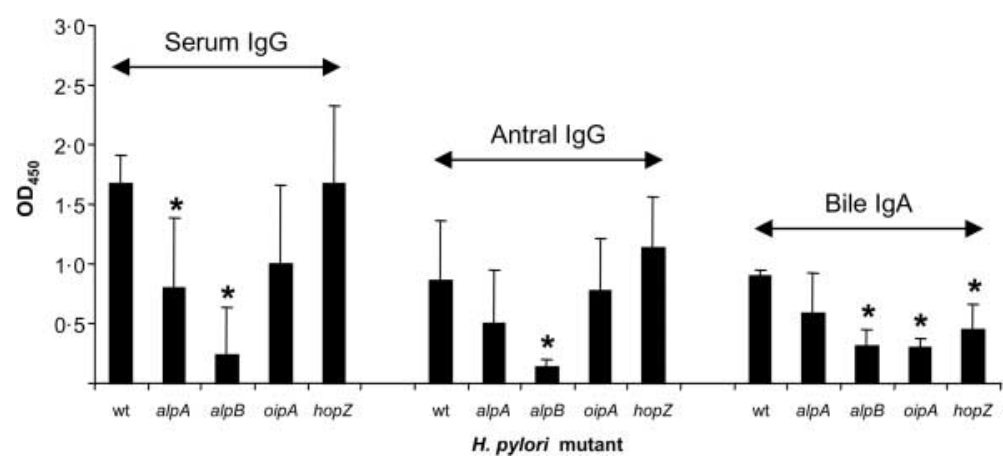

Fig. 2. Serum and local antibody responses to colonization by wild-type strain H. pylori GP15 (wt) or single OMP mutants (alpA, alpB, oipA or hopZ). Data are means of the $\mathrm{OD}_{450}$. Error bars denote SD; asterisks indicate a significant difference between the antibody response after infection by OMP mutants, compared to infection by the wild-type strain GP15 $(P<0.05)$. 
M. J. (2002). Host cell responses to genotypically similar Helicobacter pylori isolates from United States and Japan. Clin Diagn Lab Immunol 9 , $167-175$

Bergquist, C., Mattsson-Rydberg, A., Lönroth, H. \& Svennerholm, A.-M. (2000). Development of a new method for the determination of immune responses in the human stomach. J Immunol Methods 234, 51-59.

Bijlsma, J. J. E., Vandenbroucke-Grauls, C. M. J. E., Phadnis, S. H. \& Kusters, J. G. (1999). Identification of virulence genes of Helicobacter pylori by random insertion mutagenesis. Infect Immun 67, 2433-2440.

Boren, T., Falk, P., Roth, K. A., Larson, G. \& Normark, S. (1993). Attachment of Helicobacter pylori to human gastric epithelium mediated by blood group antigens. Science 262, 1892-1895.

Dubois, A., Berg, D. E., Incecik, E. T., Fiala, N., Heman-Ackah, L. M., Perez-Perez, G. I. \& Blaser, M. J. (1996). Transient and persistent experimental infection of nonhuman primates with Helicobacter pylori: implications for human disease. Infect Immun 64, 2885-2891.

Durrani, Z. \& Rijpkema, S. (2003). Orogastric vaccination of guinea pigs with Helicobacter pylori sonicate and a high dose of cholera toxin lowers the burden of infection. FEMS Immunol Med Microbiol 36, 169-173.

Eaton, K. A. \& Krakowka, S. (1994). Effect of gastric pH on ureasedependent colonization of gnotobiotic piglets by Helicobacter pylori. Infect Immun 62, 3604-3607.

Eaton, K. A., Suerbaum, S., Josenhans, C. \& Krakowka, S. (1996). Colonization of gnotobiotic piglets by Helicobacter pylori deficient in two flagellin genes. Infect Immun 64, 2445-2448.

Evans, D. J., Jr \& Evans, D. G. (2000). Helicobacter pylori adhesins: review and perspectives. Helicobacter 5, 183-195.

Guruge, J. L., Falk, P. G., Lorenz, R. G., Dans, M., Wirth, H.-P., Blaser, M. J., Berg, D. E. \& Gordon, J. I. (1998). Epithelial attachment alters the outcome of Helicobacter pylori infection. Proc Natl Acad Sci U S A 95, 3925-3930.

Hessey, S. J., Spencer, J., Wyatt, J. I., Sobala, G., Rathbone, B. J., Axon, A. T. \& Dixon, M. F. (1990). Bacterial adhesion and disease activity in Helicobacter associated chronic gastritis. Gut 31, 134-138.

Hirayama, F., Takagi, S., Yokoyama, Y., Iwao, E. \& Ikeda, Y. (1996). Establishment of gastric Helicobacter pylori infection in Mongolian gerbils. J Gastroenterol 31 (Suppl. 9), 24-28.

Home Office (1986). Guidance on the operation for the Animals (Scientific Procedures) Act 1986. London, UK: The Stationery Office.

Ilver, D., Arnqvist, A., Ogren, J. \& 7 other authors (1998). Helicobacter pylori adhesin binding fucosylated histo-blood group antigens revealed by retagging. Science $\mathbf{2 7 9}, 373-377$.

Kuipers, E. J. (1997). Helicobacter pylori and the risk and management of associated diseases: gastritis, ulcer disease, atrophic gastritis and gastric cancer. Aliment Pharmacol Ther 11 (Suppl. 1), 71-88.

Mahdavi, J., Sonden, B., Hurtig, M. \& 20 other authors (2002). Helicobacter pylori SabA adhesin in persistent infection and chronic inflammation. Science 297, 573-578.
Marchetti, M., Arico, B., Burroni, D., Figura, N., Rappuoli, R. \& Ghiara, P. (1995). Development of a mouse model of Helicobacter pylori infection that mimics human disease. Science 267, 1655-1658.

Odenbreit, S., Till, M., Hofreuter, D., Faller, G. \& Haas, R. (1999). Genetic and functional characterization of the alp $A B$ gene locus essential for the adhesion of Helicobacter pylori to human gastric tissue. Mol Microbiol 31, 1537-1548.

Odenbreit, S., Faller, G. \& Haas, R. (2002a). Role of the AlpAB proteins and lipopolysaccharide in adhesion of Helicobacter pylori to human gastric tissue. Int J Med Microbiol 292, 247-256.

Odenbreit, S., Kavermann, H., Puls, J. \& Haas, R. (2002b). CagA tyrosine phosphorylation and interleukin- 8 induction by Helicobacter pylori are independent from AlpAB, HopZ and Bab group outer membrane proteins. Int J Med Microbiol 292, 257-266.

Rijpkema, S. G., Durrani, Z., Beavan, G., Gibson, J. R., Luck, J., Owen, R. J. \& Auda, G. R. (2001). Analysis of host responses of guinea pigs during Helicobacter pylori infection. FEMS Immunol Med Microbiol 30, 151-156.

Rokbi, B., Seguin, D., Guy, B., Mazarin, V., Vidor, E., Mion, F., Cadoz, M. \& Quentin-Millet, M.-J. (2001). Assessment of Helicobacter pylori gene expression within mouse and human gastric mucosae by real-time reverse transcriptase PCR. Infect Immun 69, 4759-4766.

Sambrook, J., Fritsch, E. F. \& Maniatis, T. (1989). Molecular Cloning: a Laboratory Manual, 2nd edn. Cold Spring Harbor, NY: Cold Spring Harbor Laboratory.

Sanchez, V., Gimenez, S., Haensler, J. \& 10 other authors (2001). Formulations of single or multiple $H$. pylori antigens with DC Chol adjuvant induce protection by the systemic route in mice. Optimal prophylactic combinations are different from therapeutic ones. FEMS Immunol Med Microbiol 30, 157-165.

Shomer, N. H., Dangler, C. A., Whary, M. T. \& Fox, J. G. (1998). Experimental Helicobacter pylori infection induces antral gastritis and gastric mucosa-associated lymphoid tissue in guinea pigs. Infect Immun 66, 2614-2618.

Sturegard, E., Sjunnesson, H., Ho, B., Willen, R., Aleljung, P., Ng, H. C. \& Wadstrom, T. (1998). Severe gastritis in guinea-pigs infected with Helicobacter pylori. J Med Microbiol 47, 1123-1129.

Tomb, J.-F., White, O., Kerlavage, A. R. \& 39 other authors (1997). The complete genome sequence of the gastric pathogen Helicobacter pylori. Nature 388, 539-547.

van Vliet, A. H. M., Wooldridge, K. G. \& Ketley, J. M. (1998). Ironresponsive gene regulation in a Campylobacter jejuni fur mutant. J Bacteriol 180, 5291-5298.

Yamaoka, Y., Kita, M., Kodama, T., Imamura, S., Ohno, T., Sawai, N., Ishimaru, A., Imanishi, J. \& Graham, D. Y. (2002). Helicobacter pylori infection in mice: role of outer membrane proteins in colonization and inflammation. Gastroenterology 123, 1992-2004. 